\title{
Flux corrected transport solver for solving 1D multiphase equations for drilling applications
}

\author{
P. A. Varadarajan \& P. S. Hammond \\ Schlumberger Gould Research, Cambridge, UK
}

\begin{abstract}
A numerical solver is designed and implemented to solve a simplified set of equations modeling 1D multi-phase flow for the oil and gas drilling industry. The main motivation of this work is to capture the key features of kicks taken while drilling vertically using an oil based mud. The physical setup of a two fluid two phase (liquid and vapour phase) flow with solubility of one component is considered. A numerical method is devised to solve the system using the finite volume strategy. The difficulty in obtaining the analytical Jacobian of the fluxes leads to the decreased accuracy in capturing the contact wavefront. To address this issue a fully explicit second order numerical solver based on Flux corrected transport (FCT) is implemented. The code is validated and the physical test cases of gas injection inside the well annulus and shut-in of the well are presented. The results conclude that the FCT solver is a better and efficient method for multiphase models involving slip velocities given by algebraic relations.

Keywords: multiphase flow, kicks, drilling, explicit numerical solver, finite volume method, flux corrected transport, algebraic slip law, contact wavefronts.
\end{abstract}

\section{Introduction}

Multi-phase flows are encountered in various Engineering applications. One such scenario is the presence of gas kicks taken while drilling using oil based mud. In this work we will concentrate on the 1D fluid model that describes a two phase flow inside a vertical pipe with phases denoted by liquid phase and vapour phase that mimics the flow of drilling fluid with gas in the wellbore annulus. The model discussed is a one dimensional drift flux model consisting of two mass conservation equations and one mixture momentum equation. The liquid and the gas velocities are related by algebraic model proposed by Zuber and Findlay [1]. 
The coefficients for the slip model are obtained from the works of Johnson and Cooper [2] to predict the gas migration velocities in a realistic drilling geometry with rheological accurate fluids. The dissolution of gas in liquid is also considered, modeled by K-factor description [3]. The aim of this work is to capture the physics described by the model, efficiently using a robust and an accurate numerical scheme.

The overall report is organized in sections as follows. Section 2 describes the governing equations for a two fluid two phase flow model, listing the assumptions and its mathematical structure. Section 3 describes the FCT numerical strategy implemented to solve the model equations. Section 4 presents the solutions to a set of Riemann problems followed by the physical test cases of gas injection and shut in conditions in a vertical well. Section 5 deals with the conclusions.

\section{Governing equations}

We consider a 1D setup of a fluid flow of a two phase system with a mixture of oil and gas components. The assumptions made are

- fluid flow is inside a pipe with a constant cross sectional area A and the fluid properties are cross section averaged

- isothermal flow conditions with ideal equation of state for both the fluids

- both the fluids are compressible

- mechanical equilibrium between both the phases so that the pressure in each phase is the same along a cross section

- gas component is liquid soluble the oil is non volatile

The governing equations are a set of mass conservation equations along with the mixture momentum conservation equation. The components $U_{1}$ represents the mixture mass with $U_{2}$ the gas component mass and $U_{3}$ the mixture momentum. Writing the equation in the conservative form we have

$$
\begin{gathered}
\mathbf{U}_{t}+\mathbf{F}_{x}=\mathbf{Q} \\
\mathbf{U}=\left(\begin{array}{c}
\rho_{m} \\
\rho_{g} \alpha+(1-\alpha) \omega_{m l} \rho_{l} \\
\rho_{m} u_{m}
\end{array}\right)=\left(\begin{array}{c}
U_{1} \\
U_{2} \\
U_{3}
\end{array}\right) \\
\mathbf{F}=\left(\begin{array}{c}
U_{3} \\
\frac{U_{2} U_{3}}{U_{1}}+\alpha \rho_{g}\left(1-\frac{\alpha \rho_{g}}{\rho_{m}}\right)(v-u)\left(1-\omega_{m l}\right) \\
\frac{U_{3}^{2}}{U_{1}}+\frac{\rho_{l} \rho_{g}(1-\alpha) \alpha(u-v)^{2}}{\rho_{m}}+p \\
Q_{g} \\
Q_{g}
\end{array}\right) \\
\mathbf{Q}=\left(\begin{array}{c}
U_{1} \cos (\theta)
\end{array}\right)
\end{gathered}
$$

where $\rho_{l}$ is the liquid phase density, $\rho_{g}$ the gas phase density, $\alpha$ the vapour volume fraction, $\rho_{m}=(1-\alpha) \rho_{l}+\alpha \rho_{g}$ is the mixture density, $\omega_{g l}$ is the mass fraction of 
the gas component in the liquid phase, $u$ is the liquid phase velocity and $v$ the gas phase velocity, $u_{m}$ is the mass averaged velocity, $g$ the gravitational acceleration and $\theta$ corresponds to the orientation of the duct/pipe ( $=0$ to a vertical duct and $\theta=\pi / 2$ for a horizontal duct). $Q_{g}$ represents the source term for gas production from the formation. $p_{\text {fric }}$ represents the friction losses associated with the pipe flow. The liquid and gas phase velocities are related by algebraic slip law. The algebraic slip model is described by the relation

$$
v=C(\alpha) u_{V}+v_{s}(\alpha)
$$

where $u_{V}$ is the volume averaged velocity of the mixture with $C$ and $v_{s}$ the coefficients which are functions of the volume fraction. There is no experimental data reported for void fractions above $50 \%$ for the oil based mud scenario [2], so we have assumed the slip law in that region based on the plausible extension to the extreme scenario of $\alpha=1.0$.

The phase solubility is computed from the $\mathrm{K}$ factor model which gives the relationship between the pressure and the mixture composition in each phase. Volume filling condition is used to compute pressure once the masses of both the components are known. The molecular weight oil is taken to be $M_{l}=158$ and that of gas to be $M_{g}=16$ with critical pressure of $p_{\text {crit }}=2 \times 10^{7} \mathrm{~Pa}$. The equation of state for both the phases are modelled as

$$
\begin{aligned}
\rho_{g g} & =\rho_{g g, \text { ref }} \frac{p}{p_{\text {ref }}} \\
\rho_{g l} & =\rho_{l l}=\rho_{l l, \text { ref }}\left(1+c_{l l} \frac{p-p_{\text {ref }}}{p_{\text {ref }}}\right)
\end{aligned}
$$

where $\rho_{l l}$ represents the density of liquid component in the liquid phase,$\rho_{g l}$ the density of gas component in liquid phase and $\rho_{g g}$ the density of gas component in the vapour phase with $p_{\text {ref }}=10^{5} \mathrm{~Pa}, \rho_{\text {gg,ref }}=1 \mathrm{kgm}^{-3}, \rho_{l l, \text { ref }}=850 \mathrm{kgm}^{-3}$ and $c_{l l}=10^{-4}$. To avoid negative densities arising during numerical evaluation, the liquid densities are ramped linearly to zero for pressures below $p_{\text {ref }}$. For all the test cases presented in this work, $Q_{g}$ is assumed to be zero. The pipe friction is modelled as

$$
p_{\text {fric }}=\frac{f \rho_{m} u_{m}\left|u_{m}\right|}{\sqrt{\frac{A}{\pi}}}
$$

with $f=0.05$.

Before going into the numerical strategy of solving the governing equations, it is worth understanding the mathematical structure of the governing equations 1 . It seems prudent to work with the equations in their basic conservative form. In the absence of slip between both the phase velocities, the system of equations is strictly hyperbolic with well defined acoustic speeds and the material wave speed. By analogy with results for the system with slip velocity and without phase solubility by Romate [4], Evje and Flåtten [5], the equation 1 is hyperbolic for reasonable values of parameters. Unfortunately in the presence of inter-phase slip it is not 
easy to express the Jacobian, or eigenvalues and eigenvectors, in closed form (with or without phase solubility). This poses a challenge for designing the solver for the system of equations considered. Since we are looking at the system with 3 wave speeds the material wave front is still convective even when the system is not strictly hyperbolic.

To explore the structure of the volume fraction front we assume a mathematically simple scenario by assuming the flow to be incompressible and without phase dissolution. The transport equation for $\alpha$ is (from the gas component conservation equation)

$$
\frac{\partial \alpha}{\partial t}+\frac{\partial v \alpha}{\partial x}=0
$$

The scalar transport equation for $\alpha$ for a constant volume averaged flow rate $\left(u_{V}=\right.$ const) reduces to (from equation 5)

$$
\frac{\partial \alpha}{\partial t}+\frac{\partial F(\alpha)}{\partial x}=\frac{\partial \alpha}{\partial t}+\frac{d F(\alpha)}{d \alpha} \frac{\partial \alpha}{\partial x}=0
$$

The function $F(\alpha)$ and its derivative are plotted in the figure 1.

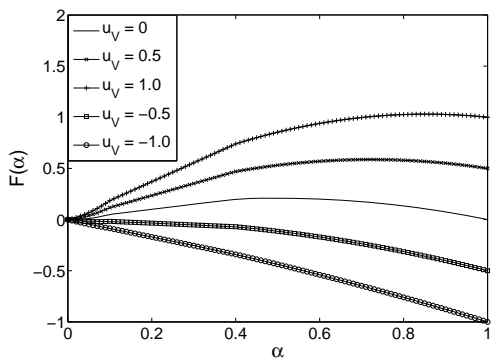

(a) Flux function

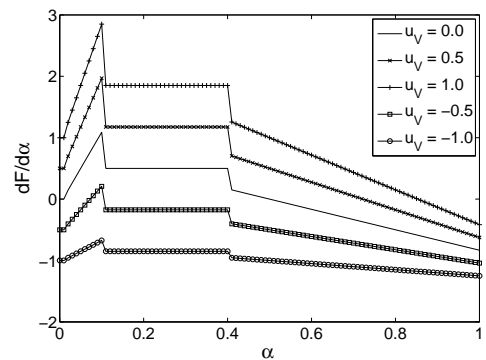

(b) convection speed of $\alpha$

Figure 1: Flux function and its derivative for scalar advection of $\alpha$.

The solution to the scalar transport equation is well known and based on the left and right states we can see how the wave structure forms. The exact solution to a Riemann problem for the scalar advection of $\alpha$ can be constructed from Oleinik's entropy condition [6]. This gives us the information that the contact wavefront is a compound wave which needs more accurate numerical methods to solve its structure.

\section{Numerical procedure: explicit second order scheme}

Explicit numerical methods are simpler to implement than implicit methods and have been implemented in this work though they suffer from serious time-step limitations due to the fast-propagating acoustic modes. We use the standard finite 
volume formulation for numerically computing the solution. The conservative variables are averaged along the cell centers $(j$-index along the $\mathrm{x}$ domain $)$ and then fluxes are computed along the interfaces. The discrete form of the conservation equation showing the update of the solution from $n$ to $n+1$ time interval is as follows

$$
U_{j}^{n+1}=U_{j}^{n}-\frac{d t}{d x}\left[F_{j+1 / 2}^{* n}-F_{j-1 / 2}^{* n}\right]+\tilde{Q}_{j} d t
$$

The task lies in accurately computing the fluxes at the interface and then integrating the source term. Integration of the source term is straight forward in our application and is discussed at the end of the section. To compute the flux at the interface we solve the Riemann problem between the adjacent cells which is the standard Godunov formulation of solving the hyperbolic equations. For the homogeneous flow without slip where the system is purely hyperbolic, the fluxes can be computed based on a more accurate solver like HLLC [7] or Roe [8] type solver. But for the system with slip, we resort to the fluxes computed from a solver which hides the Riemann problem.

In 1973 Boris and Book [9] proposed a two step blend of a first order upwind method and Lax Wendroff method calling it a Flux corrected method. The basic idea behind the method is that in a single step the flux is computed twice, one using a lower order method (generally which is diffusive in nature) and then corrected using the difference between a flux computed from the Lax Wendroff method and the first order flux as shown in equation 12. This difference in flux can be viewed as an anti diffusive flux which has to be limited to prevent oscillations. There are different ways to compute the anti diffusive (AD) flux and $\Omega$, but here we use the solution at time $n$ to compute the AD flux and the limiting variable $\Omega$. For our system of equations we chose to obtain $\Omega$ by limiting the gradients of volume fraction.

$$
\begin{aligned}
F^{*}\left(U_{L}, U_{R}\right) & =F^{*}\left(U_{L}, U_{R}\right)_{F O}+\Omega\left[F^{*}\left(U_{L}, U_{R}\right)_{L W}-F^{*}\left(U_{L}, U_{R}\right)_{F O}\right] \\
& =F^{*}\left(U_{L}, U_{R}\right)_{F O}+\Omega F^{*}\left(U_{L}, U_{R}\right)_{A D}
\end{aligned}
$$

In our computations for the first order flux, HLL solver has been implemented. The more detailed notion about the HLL solver can be found in the works of Harten, Lax and van Leer [10]. The wave speeds for the HLL solver are computed for the homogeneous setup with the eigen values being $\frac{U_{3}}{U_{1}} \pm c$, with $c$ the acoustic speed of the mixture. The important part is to compute the averaging factor $\Omega$ to limit the anti-diffusion. The limiting is required only when the solution is monotone. We have used the result for the limiting parameter $\Omega$ from the limiting done to the scalar advection equation [9].

Overall the numerical procedure is as follows, the solution at time level $n$ is updated to $n+1$ by equation 11 . The first order flux is computed by HLL scheme and second order flux is computed from Lax Wendroff scheme. Both the fluxes are averaged using the coefficient $\Omega$ obtained from the distribution of volume fraction at time step $n$. The averaged fluxes are used in 11 to the find the solution at time interval $n+1$. The source terms are integrated by averaging the conservative vector 
at time step $n$ and the value with the update of conservative vector using the first order flux at step $n+1$.

\section{Results and discussion}

Initially to validate the code for the accuracy of the model, we resort to solving Riemann type problems with gravity and friction switched off. These mathematical test cases are similar to that of shock tube problems for flow along a horizontal duct with the slip term acting as a source of momentum to make the gas escape in the positive $\mathrm{x}$ direction (based on the sign of $v_{s}$, negative direction for negative $v_{s}$ ). Two Riemann problems showing the shock-contact-shock and expansion-contactexpansion wave structures are presented. Then, two physical cases of gas kick in a vertical well and then shut in of the well are simulated and discussed.

\subsection{Riemann problems}

We present here two shock tube Riemann problems to show the capabilities of the numerical solver to predict with accuracy the wave structure. The test cases show the presence of shocks, expansion and the contact wave structure. The test case is similar to the ones reported in [11] the difference being that for the same states we have considered phase transfer along with the dynamics of the slip law. For these two Riemann problems ghost cell approach is considered with non reflective type boundary condition and the solution is presented only for times such that the waves have not reached the boundary.

\subsubsection{Two fluid expansion-contact-expansion}

The computation is performed in a tube of length $L=100 \mathrm{~m}$ with the discontinuity placed half way along the tube. The two states are given as follows

$$
\mathbf{U}_{L}=\left(\begin{array}{c}
454.915 \\
4.913082 \\
454.915 \times 1.7461
\end{array}\right) \quad \mathbf{U}_{R}=\left(\begin{array}{c}
453.197 \\
6.3901 \\
453.197 \times 24.8074
\end{array}\right)
$$

The numerical results are presented at time $t=0.4 s$ using the FCT solver with obtaining the averaging coefficient from the gradients of $\alpha$. The domain consists of 100 cells and a variable time stepping is used to compute the solution retaining the CFL number to be 0.5 computed based on the cell average values. The results show two outgoing expansion waves and a contact wave as shown in the figure 2 .

\subsubsection{Two fluid shock-contact-shock}

This test case is the mirror image of the left and right states from the previous case presented. The numerical parameters are the same as the previous case described.The solution is plotted at $t=0.5 \mathrm{~s}$ and is shown in the figure 3 . The results show two outgoing shock waves going at the acoustic speed and the contact wave getting convected with the flow. 


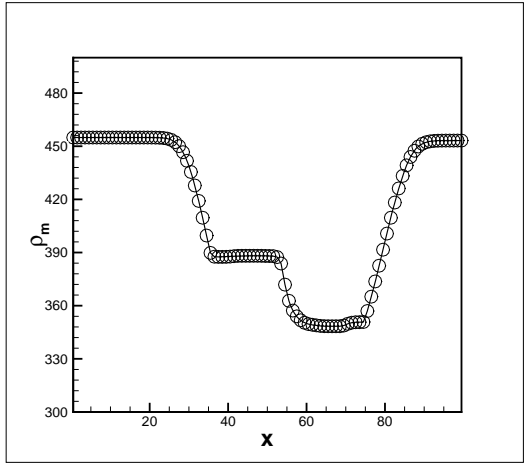

(a) average density in $\mathrm{kgm}^{-3}$

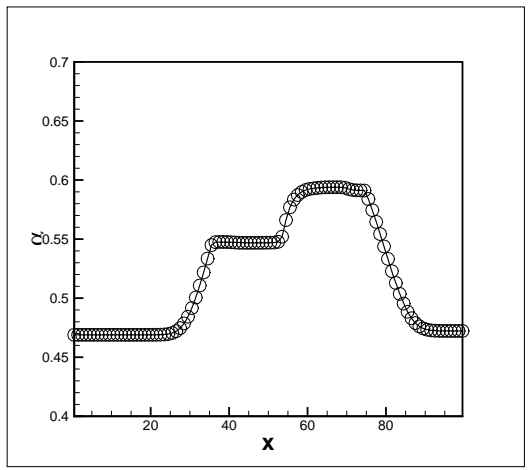

(c) vapour volume fraction

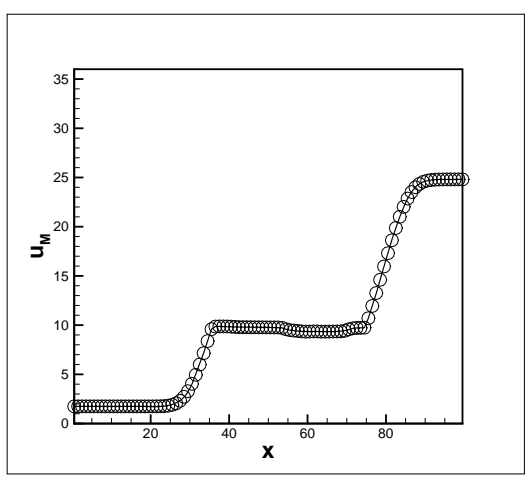

(e) Mass averaged velocity in $m s^{-1}$

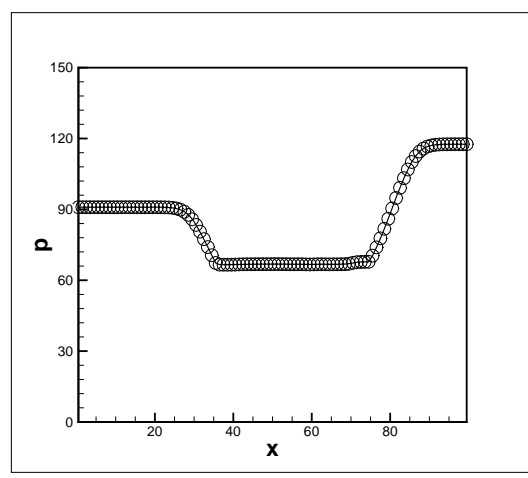

(b) pressure in $p s i$

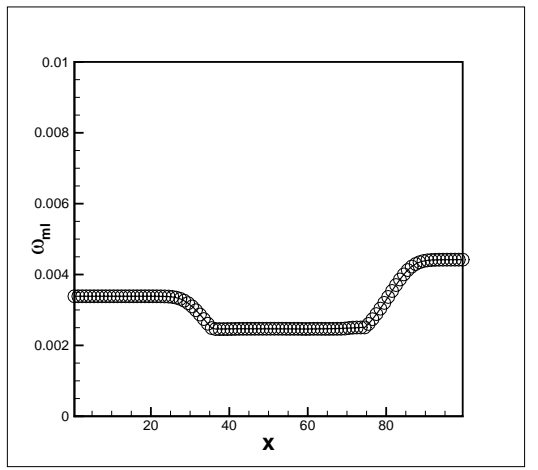

(d) gas mass fraction in liquid phase

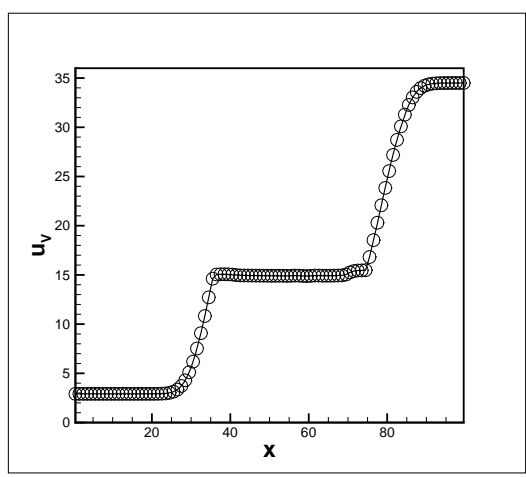

(f) volume averaged velocity $m s^{-1}$

Figure 2: Shock tube problem: two fluid expansion-contact-expansion problem. 


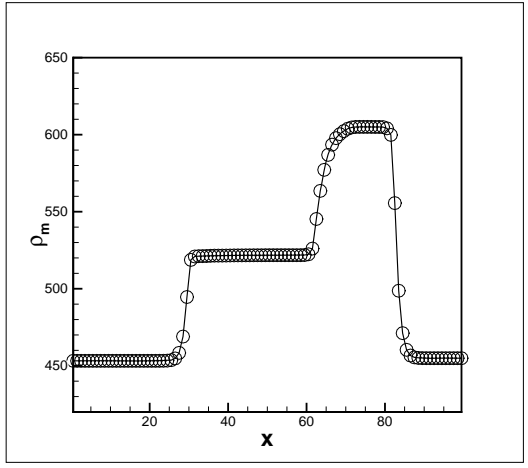

(a) average density in $\mathrm{kgm}^{-3}$

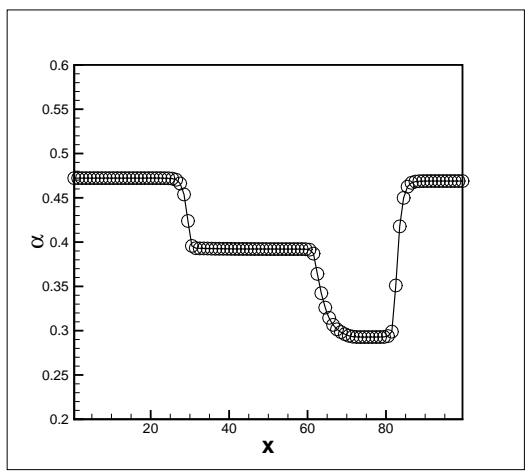

(c) vapour volume fraction

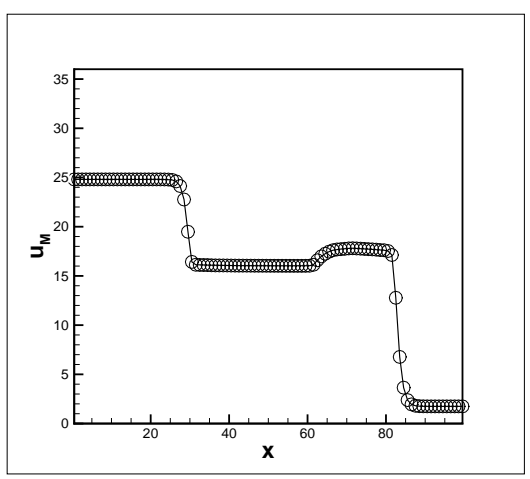

(e) Mass averaged velocity in $m s^{-1}$

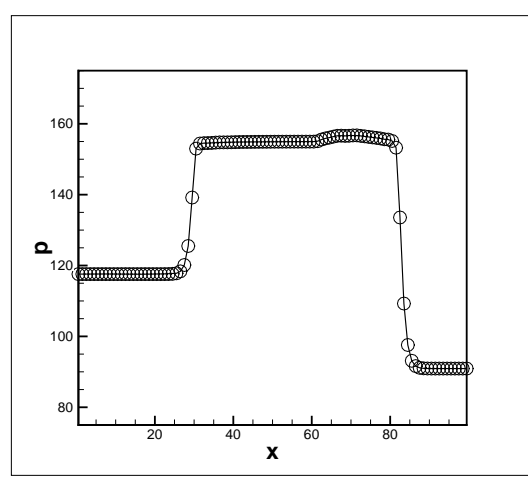

(b) pressure in psi

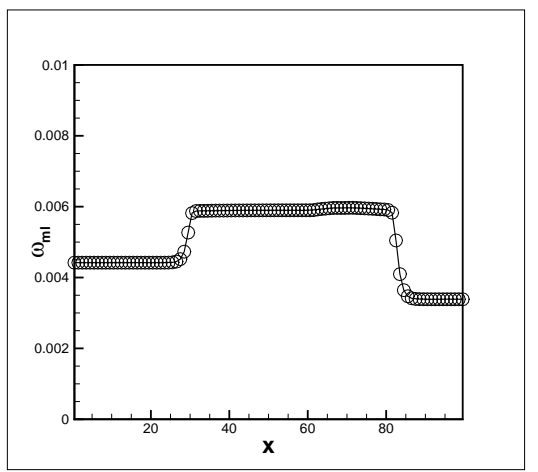

(d) gas mass fraction in liquid phase

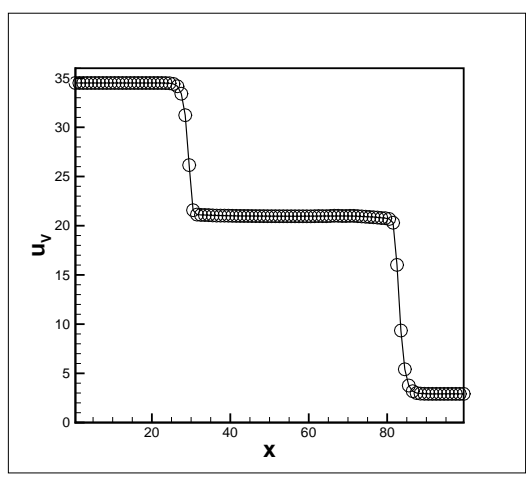

(f) volume averaged velocity $m s^{-1}$

Figure 3: Shock tube problem: two fluid shock-contact-shock problem. 


\subsection{Gas injection in a vertical well}

With the discussion of the code for the cases of flow along a horizontal section, we now present the results pertaining to gas kick from the bottom of the vertical well. Gravity is switched on and pipe friction is considered for this flow. Here we initialize a vertical tube of length $100 \mathrm{~m}$ with a standing water column. Then from the bottom of the well a mixture of gas and liquid is injected with constant flow rates. Along the ghost cell near the bottom wall the pressure is extrapolated linearly based on the slope of the adjacent two cells and the flow rates of gas and liquid are given as 99.099 and $1716.68 \mathrm{Kg} / \mathrm{s} / \mathrm{m}^{2}$ respectively. The exit pressure is maintained at 10 bar. The momentum is maintained constant for the ghost cell at the exit boundary. The solution is computed with 100 cells and CFL $=0.5$ and is plotted till $t=30 \mathrm{sec}$. The plots in figure 4 show a sharp volume fraction wavefront propagating to the top. The other variables have oscillations near the discontinuity, predominantly in the case of velocity variables and pressure.

The choice of variable to limit is $\alpha$ and no other choice gives better result than this one. When there in no phase solubility the numerical oscillations seen when $\alpha$ is limited are less, owing to the fact that $\alpha$ becomes one of the information being transported. No such clear choice of limiting variable could be deduced with the presence of phase solubility and limiting $\alpha$ gives better results compared to the other choices of the variables. It has been proved by Abgrall and Karni [12] that for any Godunov-type scheme, which is fully conservative will not be able to maintain pressure equilibrium and will develop a pressure oscillation across material fronts for multi fluid models. We feel that there is a close resemblance of this to the oscillations observed and is not very conclusive as we do not have the privilege of having an accurate first order solver in the presence of the algebraic slip model.

\subsection{Shut in condition in a vertical well: settling problem}

Once the previous case is computed with the injection of gas liquid mixture through the vertical duct both the inlet and the outlet is shut off and computationally it is prescribed at $t=30 \mathrm{sec}$. The shut in condition is implemented by prescribing the fluxes at the domain boundaries. Both the liquid and the gas velocities are prescribed for no through condition of the flow and then the pressure is linearly extrapolated at both the inlet and the exit interfaces. The intermediate transient results of the variables are plotted in figure 5 and are not equally spaced in time. The FCT solver predicts clear settling of gas at the top which were not predicted by the earlier numerical models designed. The appearance of gas bubbles sticking with the mud at the bottom is captured well (when run the code for long time) as it comes from the interesting feature of the slip law at lower volume fractions, (figure 1(b)) is captured well by the code. As stated earlier the choice of limiting with $\alpha$ resolves the sharp features in the results. Different choice of limiting has been performed and the amount of diffusion plays an important role in getting the solution especially for the gas injection and shut in test cases. Those cases are not presented in this report. 


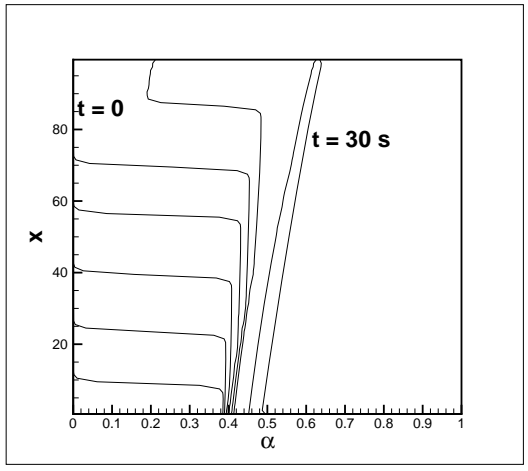

(a) vapour volume fraction

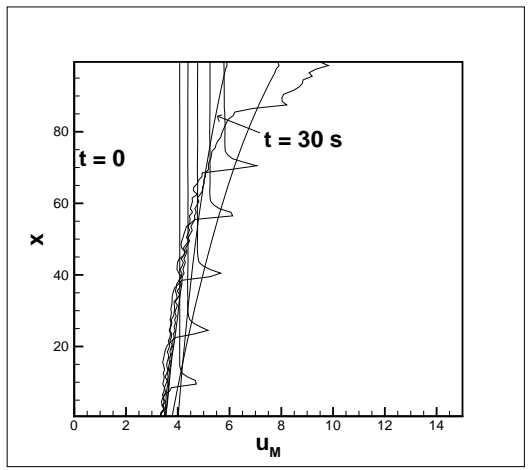

(c) Mass averaged velocity in $m s^{-1}$

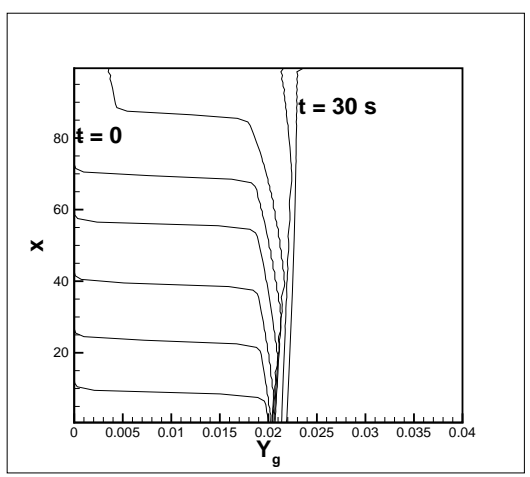

(e) Mass fraction of the gas component

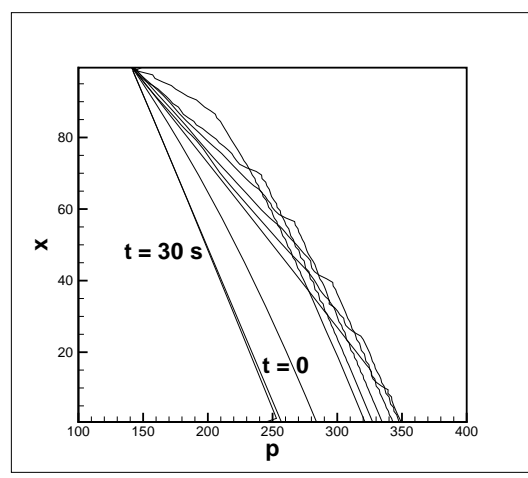

(b) pressure in $p s i$

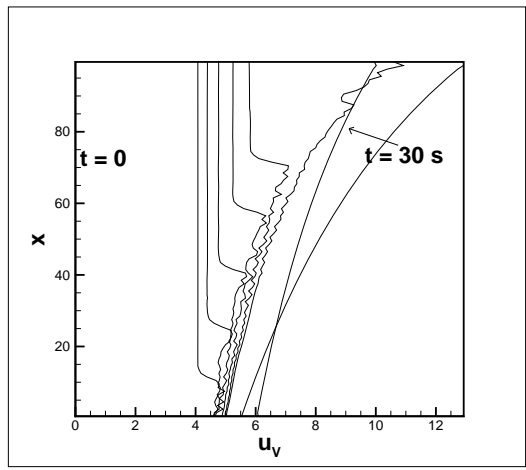

(d) volume averaged velocity $m s^{-1}$

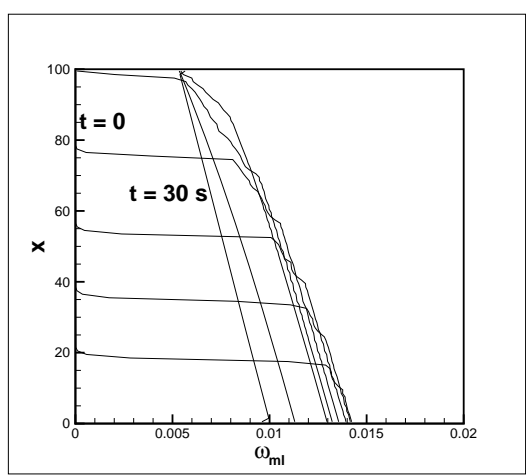

(f) gas mass fraction in liquid phase

Figure 4: FCT solution with limiting variable as the $\alpha$. 


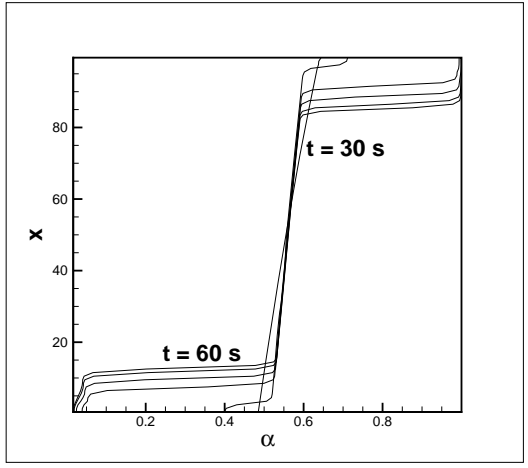

(a) vapour volume fraction

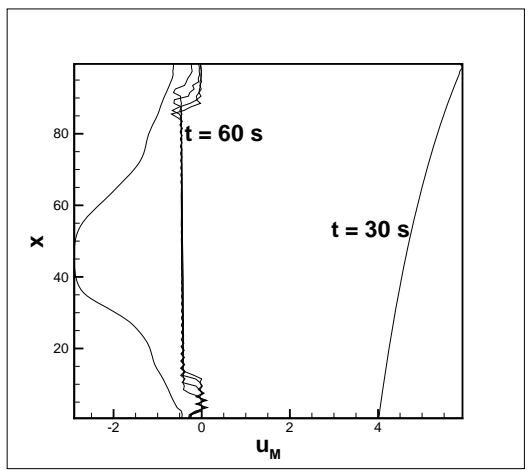

(c) Mass averaged velocity in $m s^{-1}$

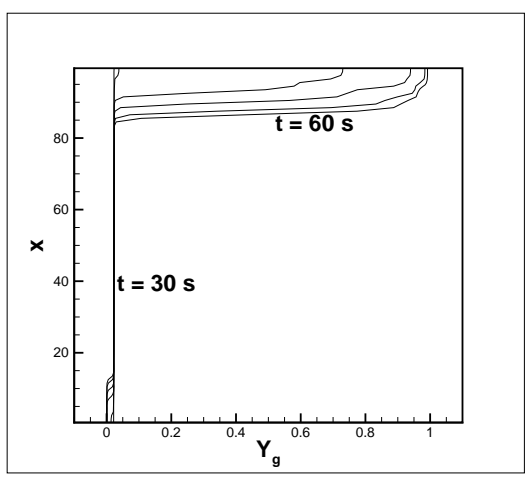

(e) Mass fraction of the gas component

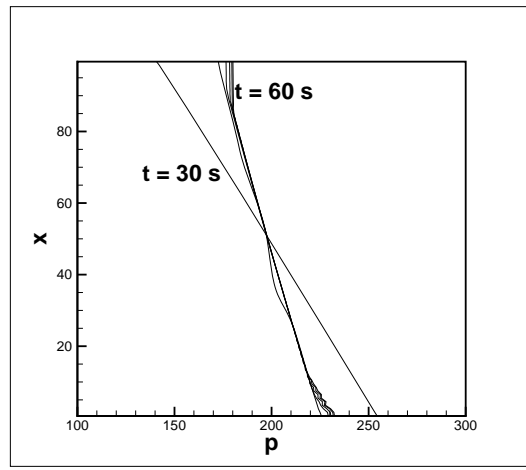

(b) pressure in psi

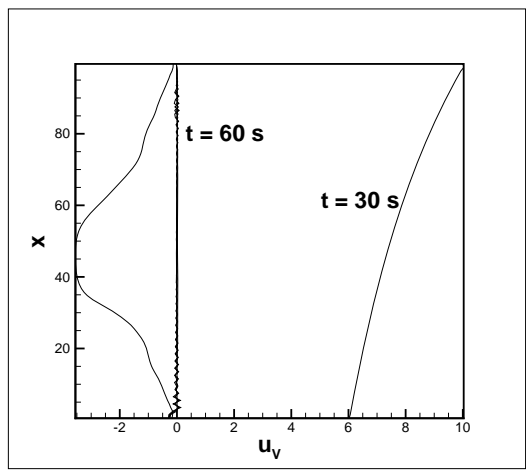

(d) volume averaged velocity $m s^{-1}$

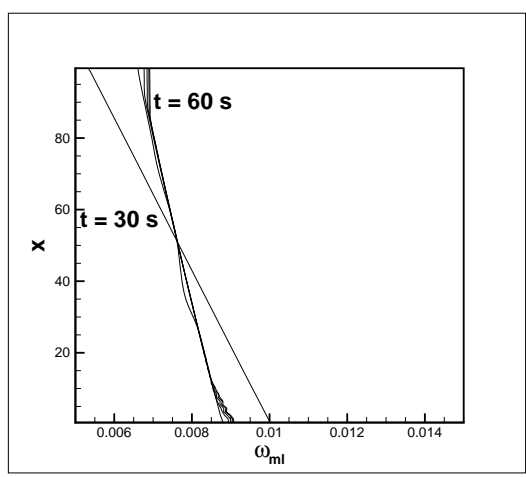

(f) gas mass fraction in liquid phase

Figure 5: FCT solution with limiting variable as the $\alpha$. 


\section{Conclusion}

The computations show that an accurate solver for the simplified model is implemented, capturing the sharp features in the volume fraction wavefront. The sedimentation case provides the proof that the current FCT solver is better in capturing the gas collection at the top and a low volume fraction of gas bubbles settling along with the liquid at the bottom given by the slip law. Thus the current solver provides less numerical diffusion in capturing sharp fronts in the solution.

Though the FCT solver is accurate in capturing the volume fraction front, it retains oscillations in the pressure and the velocity variables as discussed earlier. The drawback being the choice of variables to limit when solving for a system of equations. The solutions have less oscillations when there is no phase solubility. We insist that for practical purposes this FCT solver would work the best to capture accurate information when the slip between the phases in modeled algebraically.

\section{References}

[1] Zuber, N. \& Findlay, J., Average volumetric concentration in two-phase flow systems. J Heat Transfer, 87, pp. 453-468, 1965.

[2] Johnson, A. \& Cooper, S., Gas migration velocities during gas kicks in deviated wells. SPE Annual Technical Conference and Exhibition, 1993.

[3] McCain, W.D., The properties of petroleum fluids. PennWell Corporation, 1990.

[4] Romate, J., An approximate Riemann solver for a two-phase flow model with numerically given slip relation. Computers \& Fluids, 27(4), pp. 455-477, 1998.

[5] Evje, S. \& Flåtten, T., On the wave structure of two-phase flow models. SIAM Journal on Applied Mathematics, 67(2), pp. 487-511, 2007.

[6] Oleinik, O., Uniqueness and stability of the generalized solution of the Cauchy problem for a quasi-linear equation. Uspekhi Matematicheskikh Nauk, 14(2), pp. 165-170, 1959.

[7] Toro, E.F., Spruce, M. \& Speares, W., Restoration of the contact surface in the hll-Riemann solver. Shock Waves, 4, pp. 25-34, 1994. 10.1007/BF01414629.

[8] Roe, P., Approximate Riemann solvers, parameter vectors, and difference schemes. Journal of Computational Physics, 43(2), pp. 357-372, 1981.

[9] Boris, J.P. \& Book, D.L., Flux-corrected transport. i. shasta, a fluid transport algorithm that works. Journal of Computational Physics, 11(1), pp. 38-69, 1973.

[10] Amiram Harten, P.D.L. \& Leer, B.V., On upstream differencing and Godunov-type schemes for hyperbolic conservation laws. SIAM Review, 25, pp. 35-61, 1983.

[11] Guillard, H. \& Duval, F., A Darcy law for the drift velocity in a two-phase flow model. Journal of Computational Physics, 224(1), pp. 288-313, 2007.

[12] Abgrall, R. \& Karni, S., Computations of compressible multifluids. Journal of Computational Physics, 169(2), pp. 594-623, 2001. 